\title{
Sole Trisomy 22 Not Associated with inv(16) in Myelodysplastic Syndrome
}

\author{
Chorong Hahm, Yusun Hwang, Yeung Chul Mun ${ }^{1}$, Chu Myong Seong ${ }^{1}$, \\ Wha Soon Chung, Jungwon Huh \\ Departments of Laboratory Medicine and ${ }^{1}$ Internal Medicine, Ewha Womans University School of Medicine, Seoul, Korea
}

Trisomy 22 is closely associated with inv(16) or t(16;16) and could be a marker of cryptic rearrangement of $C B F B$ / MYH11 in acute myeloid leukemia (AML). Trisomy 22 not associated with $C B F B / M Y H 11$ rearrangement is a rare event. Here, we report a case diagnosed as refractory anemia with excess blasts-2 (RAEB-2) with sole trisomy 22 in the absence of $C B F B$ / MYH11 rearrangement. The cytogenetic study of bone marrow cells disclosed trisomy 22 in $10 \%$ of metaphase cells analyzed. The other chromosomal abnormalities were not found. Fluorescence in situ hybridization (FISH) using $C B F B / M Y H 11$ probe to detect cryptic inv(16)(p13q22) showed negative result. We also excluded rearrangements of chromosome 5, 7, 8, 20, and ETV6 by FISH. Sole trisomy 22 not associated with $\operatorname{inv}(16)$ is a true entity. (Ewha Med J 2012;35(1):62-64)

Key Words: Trisomy 22; Sole; Myelodysplastic syndrome; Auer rod

\section{Introduction}

Trisomy 22 as a sole abnormality is an uncommon cytogenetic aberration in hematological malignancies. However, it is the most common secondary chromosomal abnormality to inv(16)(p13q22) or its equivalents, suggesting that trisomy 22 could be a marker of cryptic rearrangement of inv(16)(p13q22) [1]. CBFB/MYH11 rearrangement resulting from inv(16) has been detected by RT-PCR in cases for which karyotypic analyses show sole trisomy 22 without $\operatorname{inv}(16)$ by metaphase cytogenetics.

Trisomy 22 not associated with $C B F B / M Y H 11$ re-

Received: January 13, 2012, Accepted: February 15, 2012

Corresponding author: Jungwon Huh, Department of Laboratory Medicine, Ewha Womans University School of Medicine, 911-1 Mok-dong, Yangcheon-gu, Seoul 158-710, Korea Tel: 82-2-2650-5287, Fax: 82-2-2650-5091 E-mail: JungWonH@ewha.ac.kr arrangement is a rare event. Here, we report a case diagnosed as refractory anemia with excess blasts-2 (RAEB-2) with sole trisomy 22 in the absence of CBFB/MYH11 rearrangement.

\section{Case}

An 44-year-old man was admitted to our hospital with a dyspnea. He had a history of thrombocytopenia since he was a child. Physical examinations were unremarkable. Laboratory tests were as follows: $\mathrm{Hb} 7.1$ g/dL, platelet count $35 \times 10^{9} / \mathrm{L}$, leukocyte count $7.1 \times 10^{9} / \mathrm{L}$ with $19 \%$ segmented neutrophils, $1 \%$ band neutrophil, 74\% lymphocytes, 5\% monocytes, and 1\% immature cells with Auer rods. Paroxysmal nocturnal hemoglobinuria (PNH) study using CD55 and CD59 showed negative results.

A bone marrow aspiration and biopsy showed hyper- 
cellular marrow with trilineage dysplasia. This case did not show an increased number of blasts $(0.6 \%$ of all nucleated cells), but myeloblasts included Aure rods. Proliferation of erythroid lineage was prominent $(\mathrm{M}: \mathrm{E}$ ratio= $0.2: 1$ ) and ringed-sideroblasts were not noted. There was no abnormal localization of immature precursors on biopsy and immunohistochemistry stain for CD34 and CD117 did not show the presence of an increased number of blasts. A diagnosis of RAEB-2 was made according to World Health Organization 2008 criteria [2], which classify as RAEB-2 based only on the presence of Auer rods, irrespective of the blast percentage.

The cytogenetic study of bone marrow cells disclosed trisomy 22 in $10 \%$ of metaphase cells analyzed: 47,XY, +22[2]/46,XY[18] (Fig. 1). The other chromosomal abnormalities including $\operatorname{inv}(16)$ or $\mathrm{t}(16 ; 16)$ were not found. Fluorescence in situ hybridization (FISH) using CBFB/MYH11 probe to detect cryptic inv(16)(p13q22) showed negative result. We excluded rearrangements

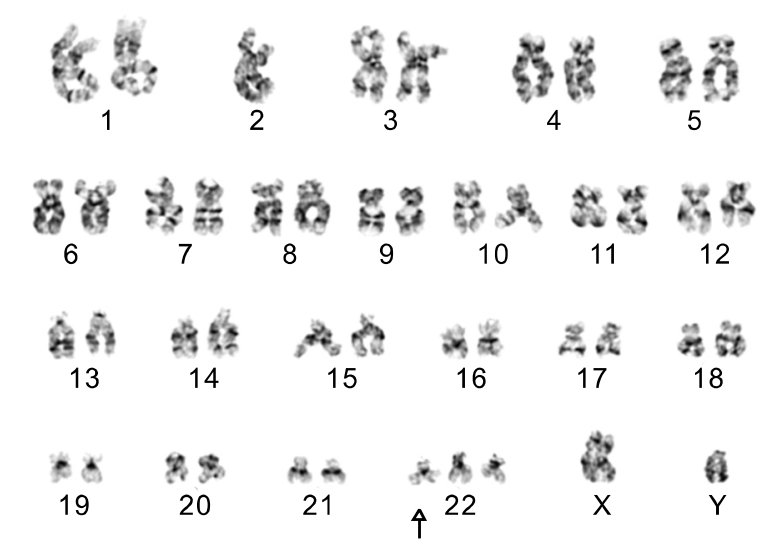

of chromosome 5, 7, 8, 20, and ETV6 by FISH using EGR1/D5S721, D5S23, CEP7/D7S486, CEP8, D20S108, and ETV6 probes.

The patient was administered azacitidine chemotherapy $(7 \mathrm{mg} / \mathrm{kg} /$ day $\times 6$ days). On the day 10 after diagnosis, a peripheral blood sample showed white blood cell counts $1.6 \times 10^{9} / \mathrm{L}$ (34\% segmented neutrophils, 58\% lymphocytes, $8 \%$ monocytes), $\mathrm{Hb} 8.3 \mathrm{~g} / \mathrm{dL}$ and platelet counts $112 \times 10^{9} / \mathrm{L}$. Immature cell was not noted.

\section{Discussion}

The present study confirmed sole trisomy 22 not associated with inv(16) by FISH using $C B F B / M Y H 11$ probe in a myelodysplastic syndrome (MDS) patient (RAEB-2). It is important to distinguish between true sole trisomy 22 and trisomy 22 associated with inv(16). When trisomy 22 is present as the secondary karyotypic abnormality of inv(16) or its equivalents, the final diagnosis should be changed to acute myeloid leukemia (AML) with inv(16), irrespective of blast cell counts, according to World Health Organization 2008 classification [2].

Previously reported cases with sole trisomy 22 not associated with inv(16) were summarized in Table 1 $[3,4]$. Taken together, sole trisomy 22 may also occur apparently in the absence of inv(16), although trisomy 22 is closely associated with $\operatorname{inv}(16)$ or its equivalents. The molecular genetic consequences and clinical impact of sole trisomy 22 remains to be elucidated.

In summary, sole trisomy 22 not associated with $\operatorname{inv}(16)$ could be a true entity. It is necessary to exclude the presence of a hidden, cryptic inv(16) by means of molecular approaches such as RT-PCR or FISH when trisomy 22 is the sole abnormality.

Fig. 1. G-banded karyotype showing $47, X Y,+22$.

Table 1. Reported cases with trisomy 22 as the sole karyotypic abnormality

\begin{tabular}{cclccl}
\hline Case no. & Sex/Age & Diagnosis & Cytogenetics & CBFB/MYH11 & Reference \\
\hline 1 & $\mathrm{~F} / 89$ & RAEB-1 & $47, \mathrm{XX},+22[16] / 46, \mathrm{XX}[4]$ & Negative & Takahashi et al. [3] \\
2 & $\mathrm{~F} / 30$ & AML $(\mathrm{M} 5 \mathrm{~b})$ & $47, \mathrm{XX},+22[3] / 46, \mathrm{XX}[17]$ & Negative & Gozzetti et al. [4] \\
3 & $\mathrm{M} / 55$ & RAEB-2 & $47, \mathrm{XY},+22[2] / 46, \mathrm{XY}[18]$ & Negative & This case \\
\hline
\end{tabular}

RAEB, refractory anemia with excess blasts; $A M L$, acute myeloid leukemia. 


\section{References}

1. Bilhou-Nabera C. del(20q) in myeloid malignancies. Huret JL, editor. Atlas of genetics and cytogenetics in oncology and haematology [Internet]. Poitiers: Genetics DIM, University Hospital; 2000 [cited 2012 Feb 10]. Available from: http://AtlasGeneticsOncology.org/Anomalies/del20qID1040.html.

2. Swerdlow SH, Campo E, Harris NL, Jaffe ES, Pileri $\mathrm{SA}$, Stein $\mathrm{H}$, et al. WHO classification of tumours of haematopoietic and lymphoid tissues. 4th ed. Lyon: International Agency for Research on Cancer; 2008.

3. Takahashi T, Harada S, Oki M, Yoshimoto M, Tsujisaki M. Trisomy 22 as the sole karyotypic abnormality in myelodysplastic syndrome. Int J Hematol 2005;81: 269-270.

4. Gozzetti A, Calabrese S, Crupi R, Tozzuoli D, Bocchia M, Fabbri A. Trisomy 22 as sole cytogenetic abnormality in acute monoblastic leukemia (M5b). Cancer Genet Cytogenet 2006;169:86. 